\title{
BIM AND MOTIVATION SELF-REGULATION STRATEGIES
} Conference of the Iberoamerican Society of Digital Graphics Medellin | Colombia

\author{
Luciano de Vasconcellos \\ Univ. Federal de Pelotas | Brasil | arqvasconcellos@yahoo.com.br \\ Lourdes Maria Bragagnolo Frison \\ Univ. Federal de Pelotas | Brasil | frisonlourdes@gmail.com \\ Eduardo Grala da Cunha \\ Univ. Federal de Pelotas | Brasil | eduardogralacunha@yahoo.com.br
}

\begin{abstract}
We investigated the contributions of BIM in the light of the Self-Regulatory Learning construct, to understand how academics mobilize strategies to become more autonomous and capable of their own learning. It was investigated how can BIM technology software brings contributions to the development of self-regulatory strategies by undergraduate students. The research was developed first performing a semi-structured interview. Then the collected data were submitted to the content analysis technique and two categories emerged: a) the potentialities of BIM technology use b) contributions of BIM technology in the use of selfregulatory strategies. The findings of this research reveal the role of BIM to support student's self-regulatory strategies.
\end{abstract}

Keywords: Self-Regulated Learning; Socially Shared, BIM; Architecture; Teaching

\section{INTRODUÇÃO}

A autorregulação da aprendizagem envolve uma capacidade que o ser humano tem de autorregular seus comportamentos, pensamentos, sentimentos com o objetivo de atingir uma meta de aprendizagem estabelecida por aquele que quer aprender (Avila, 2017). A importância do emprego adequado e correto de estratégias autorregulatórias por aprendizes escolares, desde a escola primária até o ensino universitário, se deve ao fato de estar diretamente vinculada ao desempenho acadêmico (Butler, Schnellert, \& Perry, 2017). De acordo com Veiga Simão e Frison (2013), temos o entendimento de que o ser humano tem a capacidade para autorregular sua aprendizagem, sendo esta uma de suas aptidões mais importantes. Não se trata de uma aptidão que algumas pessoas têm e outras não, porque a autorregulação precisa ser aprendida. Muitos estudantes apresentam características particulares e diferentes em relação à capacidade de se autorregular, porém o controle e a tomada de decisão são princípios fundamentais para autorregular-se.

\section{AUTORREGULAÇÃO DA APRENDIZAGEM - ARA}

Escolhemos como amparo teórico deste trabalho o modelo de Zimmerman (2013) que, ao investigar sobre autorregulação da aprendizagem, afirmou que ela não é uma habilidade, não é um comportamento e não é uma autoconfiança. Para alcançar a autorregulação, "os estudantes devem perceber falhas em sua aproximação inicial, encontrar formas de corrigi-las estrategicamente, e exercer o esforço necessário para eventualmente ter sucesso" (Zimmerman B. J., 2013, p. 137).
Cabe destacar que a autorregulação além de ser um processo cíclico e multidimensional, inclui fatores psicológicos, externos, internos que tornam possível ao indivíduo dirigir seu comportamento em função de metas e de aspirações pessoais e das exigências do meio (Bandura, 1999). O meio, contudo, não é uma entidade monolítica.

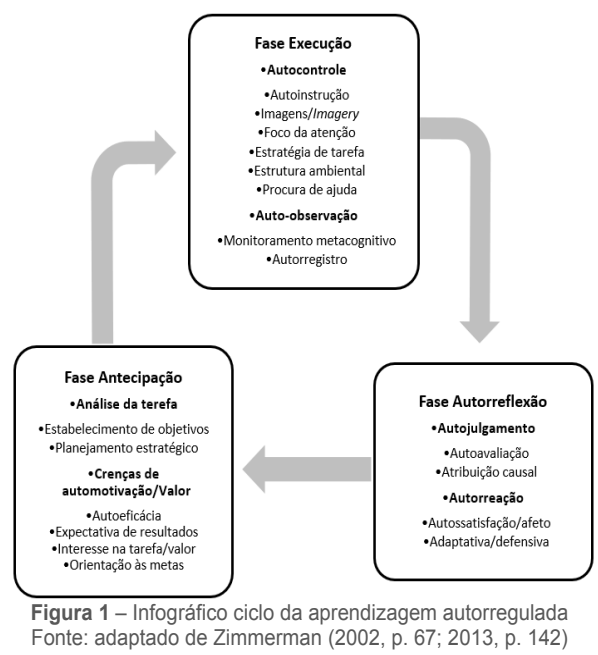

A Autorregulação da Aprendizagem é considerada um processo multidimensional porque envolve as dimensões cognitiva, metacognitiva, motivacional, comportamental, emocional e contextual, sendo elas destacadas em vários modelos, mesmo que um ou outro possa ter um determinado foco, por exemplo a motivacional (Rosário, et al., 2014). Fundamentalmente o que as pesquisas no campo da autorregulação esperam responder, é como um 
estudante mobiliza estratégias, para que possam potencializar processos específicos de aprendizagem, aumentando o nível de autoconfiança e de crenças motivacionais. $\mathrm{Na}$ figura 1 apresentamos o ciclo autorregulatório (Zimmerman B. J., 2002; 2013) com suas fases, processos e subprocessos.

Zimmerman (2013) descreve que, para capturar de forma confiável as estratégias autorregulatórias dos estudantes, desenvolveu em parceria com Martinez-Pons (1990), uma entrevista estruturada denominada SRLIS - Self-Regulated Learning Interview Scale e, com base nas respostas dadas pelos estudantes, codificaram quinze categorias de estratégias autorregulatórias.

Existem várias classificações de estratégias de aprendizagem desenvolvidas por diversos autores, mas as mais recorrentes encontradas na literatura são as classificações de: estratégias cognitivas e metacognitivas (Boruchovitch, 1999; Ximenes dos Santos \& Boruchovitch, 2011). As estratégias cognitivas são as que os aprendizes adotam de forma intencional ou consciente, tendo sobre elas um controle relativo na busca por melhorar o desempenho da assimilação, internalização, consolidação e a transferência de conhecimentos ou habilidades (Santos \& Alliprandini, 2018). As estratégias metacognitivas são os procedimentos que os aprendizes usam enquanto planejam, monitoram e regulam seus próprios pensamentos (Boruchovitch, 1999; 2014).

\section{ARA E MOTIVAÇÃO}

Uma das dimensões da autorregulação da aprendizagem é a motivação para a realização da tarefa, o que contribui para evitar distratores e superar o insucesso acadêmico. 0 insucesso pode estar ligado à falta de autorregulação, procrastinação, baixos níveis de aprendizagem e desempenho, sendo que os professores precisam ajudar os aprendizes ensinando estratégias de autorregulação para a aprendizagem específica de cada área do conhecimento (Pedersen \& Polydoro, 2017). Embora possa parecer uma questão comum aos pesquisadores em educação que fatores cognitivos e metacognitivos sejam preditores do sucesso acadêmico, a perspectiva da autorregulação também destaca a importância dos fatores motivacionais e comportamentais. A teoria de autorregulação procura entender as questões sobre onde, como e porque alguns estudantes se autorregulam e outros não (Zimmerman B. J., 2011). Ao investigar o "porquê" colocamos o foco da investigação nos aspectos motivacionais envolvidos na autorregulação da aprendizagem. Isso nos mostrará se o estudante demonstra iniciativa pessoal, perseverança e habilidade adaptativa. Autores como Wolters (2011), Paulino e Lopes da Silva (2012), Paulino, Sá e Lopes da Silva (2016) destacam a importância dos fatores relativos ao aspecto motivacional da autorregulação da aprendizagem, explicando que a capacidade dos aprendizes em regular sua motivação impacta no desempenho.

\section{BIM E MOTIVAÇÃO DA ARA}

Autores pontuam que a tecnologia BIM representa uma mudança de paradigma no campo do projeto de arquitetura, pois representa uma troca de crenças e técnicas que são abandonadas ou substituídas (Checcucci, Pereira, \& Amorim, 2013; Godoy, Cardoso, \& Borges, 2013; Wu \& Issa, 2013; Ruschel R. C., 2014). Com o uso dessa tecnologia, "um modelo virtual preciso de uma edificação é construído de forma digital" (Eastman, Teicholz, Sacks, \& Liston, 2014, p. 1), não necessitando mais de que se trabalhe com os abstratos fatiamentos do projeto por planos secantes. Um dos principais paradigmas a serem mudados com o uso da tecnologia é a redistribuição dos esforços no processo de projeto, o qual deixa de ter a maior carga de trabalho focada na documentação e apresentação do objeto e passa a dar ênfase maior na fase de concepção. Outra questão-chave sobre o BIM é o suporte à integração e ao feedback para a tomada de decisão na fase de concepção do projeto (Eastman, Teicholz, Sacks, \& Liston, 2014), fator importante do ciclo autorregulatório por atuar diretamente na fase de autorreflexão ao contribuir com a autoavaliação.

É necessário que as ações do processo em BIM sejam planejadas com as adequadas estratégias motivacionais e volitivas para tarefas de projetos com o emprego dessa tecnologia. A Curva de MacLeamy (figura 2) demonstra que quanto mais tarde ocorrerem alterações no processo, maior será custo para o projeto e, no foco deste trabalho com relação ao custo da tarefa. Com relação à motivação para autorregulação, estudantes tendem a não perseverar quando avaliam a tarefa como sendo de alto custo (Zimmerman B. J., 2002).

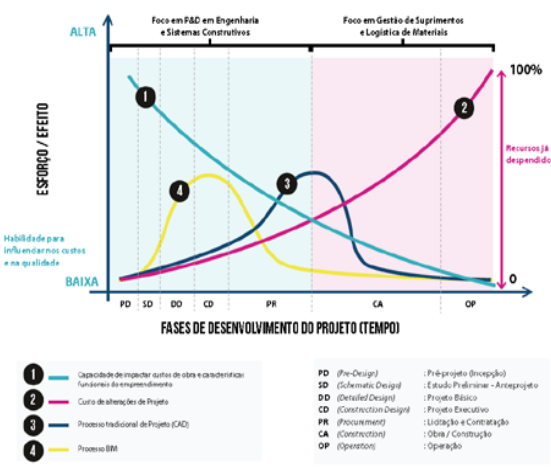

Figura 2 - Curva de MacLeamy

Fonte: Câmara Brasileira da Indústria da Construção (2016)

Martinez (2000) observa que o processo de projeto pode sim se beneficiar da computação gráfica e entendemos que, embora o autor não mencione, era compreendida naquele momento como CAD por uma questão de temporalidade. Nas palavras de Martínez (2000, p. 46), o novo recurso proporciona a "aceleração do processo de tentativa e erro na invenção arquitetônica", e, por aceitar várias alterações como um "escravo", dessas transformações "extrai consequências para exibi-las ao projetista, aumentando assim seu poder". Entendemos que o poder referido pelo autor é o poder de projetar e dar soluções, sendo assim, contribuindo para o ciclo autorregulatório. Martinez (2000) também destaca que o projeto resulta da produção de um conjunto de especificações e representações que permite construir o objeto idealizado.

Nossa convicção para ações de ensino se baseia no entendimento de que, para um programa de instrução ser bem-sucedido, ele deve levar em conta o contexto de sala de aula e, com isso, encorajar estudantes a serem 
estratégicos (Souza, 2010). Considerando que a maior problemática das práticas de ensino seja a projetual, e que crenças como a necessidade de ter-se talento ou dom, remetem à ideia de que não seja possível aprender ou ensinar (Jantzen, 2009). Então, o campo das crenças de autoeficácia e das motivações dos estudantes fica muito afetado, principalmente quando se trata de estudantes inexperientes.

\section{BIM EM CONTEXTOS EDUCACIONAIS}

Autores pesquisadores da área têm focado seus estudos sobre BIM na graduação, havendo uma tendência de aumento nos estudos com pós-graduação em anos mais recentes (Adamu \& Thorpe, 2016; Machado, Ruschel, \& Scheer, 2016). Há um maior interesse na forma como o BIM é inserido nas grades curriculares seguido tanto pela avaliação dos efeitos dessa inserção sobre a aprendizagem quanto pela preocupação sobre as estratégias pedagógicas que devem ser empregadas, ou então, fazem relatos de casos de sucesso nesse campo (Barison \& Santos, 2013; Ruschel, Andrade, \& Morais, 2013; Delatorre, Pereira, \& Miotto, 2015; Vinšová, Achten, \& Matějovská, 2015). Estudos empíricos ou observacionais têm prevalecido como método de avaliação dos efeitos de aprendizagem sendo que a modalidade foi escolhida por autores ao investigar BIM e seus efeitos pedagógicos (Rêgo \& Carreiro, 2015; Adamu \& Thorpe, 2016).

$\mathrm{Na}$ busca pela adequação, com qual ferramenta da tecnologia BIM seria conveniente abordar as questões relativas aos objetivos deste trabalho, foram encontradas com relevância a utilização das ferramentas que fazem parte da suíte de programas da Autodesk ${ }^{\odot}$, principalmente ○ Revit Architecture ${ }^{\circledR}$ (Arayici, Coates, Koskela, \& Kagioglou, 2011; Ambrose, 2012; Marques, 2013; Checcucci, 2014; Nome \& Queiroz, 2014; Ruschel R. C., 2014; Romcy, Tinoco, \& Cardoso, 2015; Adamu \& Thorpe, 2016; Brígitte \& Ruschel, 2016). O foco do uso desse software foi no desenvolvimento das habilidades de "BIM 1.0", dando aos estudantes as bases de modelagem e documentação dos projetos arquitetônicos.

Segundo Flório (2007), o planejamento da ordem de utilização dos recursos de expressão determinará as ações cognitivas no ato de projetar, indicando que, ao mesmo tempo em que o estudante reestrutura a forma de conceber o problema, inventa experimentos para testar suas novas compreensões, e nessa situação o BIM contribui com um feedback sobre o impacto de cada ação tomada pelo estudante, permitindo a ele retornar a planejar e executar. Compreende-se implícito na argumentação do autor o ciclo regulatório de aprendizagem. Nesse contexto "a adoção do BIM em disciplinas isoladas pode ser considerada inapropriada" (Ruschel R. C., 2014, p. 5); logo, entendemos que o estudante deve estar capacitado, não apenas no tópico desenvolvido na disciplina de BIM, mas também deve desenvolver estratégias de aprendizagem autorregulada capazes de lhe dar suporte em toda a contínua aprendizagem e atualização que o BIM requer.

Entre os principais desafios encontrados por pesquisadores na implementação da tecnologia BIM destacam-se as questões culturais, metodológicas e de aprendizagem (Moura, Giacaglia, \& Lara, 2013; Rêgo \& Carreiro, 2015; Romcy, Tinoco, \& Cardoso, 2015). Soma- se a esses desafios o fato de a maioria dos estudantes matriculados nas fases iniciais dos cursos, e até mesmo estudantes de fases intermediárias, não terem tido nenhuma exposição anterior ao software BIM. Fator relevante é a necessidade de o professor ser também um aprendiz autorregulado, pois ele "precisa planejar adequadamente (dentro de todo este cenário) para introduzir o pacote de software, especialmente se é a primeira vez que os estudantes são expostos a ele". (Palomera-Arias \& Liu, 2016).

Entendemos que este estudo aprofunda as investigações com respeito às relações entre um sistema sociotécnico (Marques, 2013, p. 90), o BIM, e a aprendizagem autorregulada com base na teoria social cognitiva de Bandura (1999), ao investigar, com ênfase nos processos autorregulatórios para aprender, as dimensões cognitivas, metacognitivas, motivacionais e sociais.

\section{METODOLOGIA}

Optamos por uma metodologia de pesquisa qualitativa que, segundo Moraes (2004), permite aprofundar a compreensão dos fenômenos investigados, a partir de uma análise rigorosa e criteriosa sobre o objeto específico do estudo. Entendemos que, com a modalidade de pesquisa qualitativa, não teremos a preocupação de levar em consideração questões mensuráveis ou examináveis experimentalmente (Denzin \& Lincon, 2003). Mapeamos os processos e as estratégias utilizadas por estudantes do curso de Arquitetura e Urbanismo por meio de um estudo de caso, realizando uma intervenção, coletando instrumentos que nos revelaram como eles agem ao utilizarem a ferramenta BIM, ancorada em estratégias autorregulatórias

Segundo Amado (2014), as investigações qualitativas procuram o que dá sentido e como dá sentido para os sujeitos investigados. Esta modalidade de pesquisa possibilitou-nos estudar como e por que ocorre o fato, procurando encontrar o seu sentido e entender o significado que as pessoas dão a eles (Chizzotti, 2003). O autor, define pesquisa qualitativa quando há uma troca entre as "pessoas, fatos e locais que constituem objetos de pesquisa" para, posteriormente, interpretar em um texto escrito "os significados patentes ou ocultos do seu objeto de pesquisa" (Chizzotti, 2003, p. 221). Elegemos a modalidade de investigação qualitativa dada a necessidade de interpretação dos dados que foram coletados, bem como, a possibilidade de explorar a riqueza em termos de sua interpretação, considerando também a importância do papel do investigador neste tipo de pesquisa.

Destacamos, desta forma, que as pesquisas qualitativas em educação são bastante complexas, porque lidam diretamente com o ser humano, em diversas dimensões: espiritual, moral, cognitiva, social, cultural, vocacional, artística, física (Amado, 2014) e, no caso desta pesquisa, porque busca investigar junto a estudantes de graduação do curso de Arquitetura e Urbanismo se, ao utilizarem a tecnologia BIM, desenvolvem estratégias para autorregulação da aprendizagem. Para realizar a pesquisa, conforme sugere a metodologia qualitativa, optamos por desenvolvê-la por meio de um estudo de caso, entendendo que o estudo de caso contribui para 
investigar "fenômenos contemporâneos em profundidade e em seu contexto da vida real, especialmente quando os limites entre o fenômeno e o contexto não estão claramente evidentes" (Yin, 2014, p. 39).

As estratégias de obtenção da amostragem intencional também foram extensivamente estudadas durante a elaboração da pesquisa, uma vez que não se trata de amostragem probabilística voltada às inferências estatísticas sendo, ao contrário, uma amostra que buscou evidenciar de modo intencional com um grupo de estudantes o fato ocorrido naquele contexto, considerando o problema da pesquisa (Creswell, 2010). Nesta pesquisa, dadas as características de cada uma das etapas planejamos instrumentos de coleta de dados, roteiros de entrevista, formulários e escalas em papel para serem preenchidos presencialmente, como também formulários eletrônicos para a coleta de dados online. Além disso, realizamos uma intervenção na qual foram realizadas oficinas, seguidas de entrevistas semiestruturadas, gravadas e transcritas para posterior análise dos dados.

\section{PARTICIPANTES}

Não foi feita nenhuma escolha intencional quanto ao número de participantes, apenas em todas as etapas foram convidados estudantes que estivessem interessados em participar do estudo proposto por meio de formulários eletrônicos, redes sociais, página do curso, murais etc. Obviamente que o ideal seria ter o mesmo número de estudantes em cada uma das etapas, mas isso não foi possível. Com base nos estudos de Florio (2007), Godoy; Cardoso e Borges (2013) e Ledo e Pereira (2014), os quais trabalharam com estudos de caso com número de participantes semelhantes, entendemos que, com o grupo que tivemos, foi possível atingir os objetivos propostos para a pesquisa.

\section{COLETAS DE DADOS}

Os dados foram coletados em três etapas. A primeira investigando a realidade contextual dos estudantes por meio de uma pesquisa com um survey, levando em conta que se trata de uma metodologia adequada para buscar informações diretamente com o grupo de interesse. Para a coleta de dados desta etapa, organizamos e aplicamos um instrumento que continha questões relacionadas às contribuições da modelagem arquitetônica em software de tecnologia BIM, verificando o emprego de estratégias autorregulatórias na elaboração de projetos arquitetônicos. A escolha dos temas das seções do instrumento teve como guia pesquisas que investigaram a implementação ou os resultados na área da tecnologia BIM. (Kitchenham \& Pfleeger, 2002). Conforme preconiza a teoria, a coleta de dados com a utilização de questionários, tem como vantagem que estes contribuem significativamente para a realização de estudos exploratórios que buscam o levantamento de uma amostra ou de uma população, mas sem descartar a possibilidade de entrevistas que serão posteriormente explicadas (Silveira \& Córdova, 2009).

A segunda coleta de dados foi realizada por meio de uma intervenção pedagógica, realizada contendo 20 oficinas temáticas (ver quadro 1quadro 2), de 2 horas perfazendo um total de $40 \mathrm{~h}$, sobre as estratégias de autorregulação da aprendizagem utilizadas para a aprendizagem da tecnologia BIM. As oficinas fizeram parte de um projeto de ensino institucional, no qual se inscreveram os estudantes interessados no assunto. Não foi imposto um número de vagas sendo apenas solicitado que os interessados estivessem regularmente matriculados no Curso de Graduação em Arquitetura e Urbanismo e cursando ou já ter cursado disciplinas que envolvessem a prática do desenvolvimento de projetos arquitetônicos.

Quadro 1 - Resumo das oficinas ministradas

\begin{tabular}{|c|c|c|}
\hline $\begin{array}{c}\text { Número da } \\
\text { oficina }\end{array}$ & O que? & Como? \\
\hline 1 & $\begin{array}{l}\text { Aprender o que é } \\
\text { BIM }\end{array}$ & $\begin{array}{l}\text { Leitura de textos, explicação do } \\
\text { conteúdo, fichas/resumos no } \\
\text { AVA. }\end{array}$ \\
\hline 2 & $\begin{array}{l}\text { Como se utiliza o } \\
\text { BIM }\end{array}$ & $\begin{array}{l}\text { Aulas expositivas; Busca de } \\
\text { informações; Modelamento }\end{array}$ \\
\hline 3 & $\begin{array}{l}\text { Aprender o que é } \\
\text { ARA e as fases } \\
\text { cíclicas }\end{array}$ & $\begin{array}{l}\text { Apresentação e discussão } \\
\text { sobre as fases cíclicas } \\
\text { Dimensões da ARA }\end{array}$ \\
\hline 4 & $\begin{array}{|lr|}\text { Estratégias de } & \text { aprendizagem } \\
\text { como geri-las } & \end{array}$ & 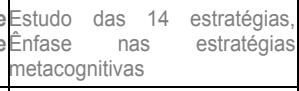 \\
\hline 5 & Gestão do tempo & $\begin{array}{l}\text { Pomodoro - o que fazer para } \\
\text { não procrastinar }\end{array}$ \\
\hline 6 & $\begin{array}{l}\text { Trabalho } \\
\text { colaborativo }\end{array}$ & $\begin{array}{l}\text { Propostas de tarefas a serem } \\
\text { realizadas em pares - ajuda } \\
\text { social }\end{array}$ \\
\hline $7,8,9$ & \begin{tabular}{|l|} 
Tarefas \\
distância com \\
monitoramento.
\end{tabular} & $\begin{array}{l}\text { Modelagem de edificação, } \\
\text { controle do tempo e fontes de } \\
\text { informação pesquisadas }\end{array}$ \\
\hline 10 & $\begin{array}{l}\text { Orientações } \\
\text { individuais }\end{array}$ & $\begin{array}{l}\text { Atividades de pequenos grupos, } \\
\text { trabalho em pares e avaliação e } \\
\text { planejamento das próximas } \\
\text { etapas }\end{array}$ \\
\hline 11 & $\begin{array}{l}\text { Cumprimento de } \\
\text { tarefas no dia } \\
\text { marcado }\end{array}$ & \begin{tabular}{|l|l|} 
Revisão de tarefas e \\
orientações individuais sobre a \\
modelagem de edificação de \\
acordo com modelo
\end{tabular} \\
\hline $\begin{array}{l}12,13,14 \\
15,16,17\end{array}$ & $\begin{array}{l}\text { Sessões } \\
\text { intermediárias }\end{array}$ & $\begin{array}{l}\text { Revisão no AVA de tarefas e } \\
\text { orientações individuais } \\
\text { adequação ao modelo; } \\
\text { avaliação das estratégias de } \\
\text { autocontrole e autorregulação } \\
\end{array}$ \\
\hline $18,19,20$ & \begin{tabular}{|l|} 
Autoavaliação \\
utilizando BIM e \\
ARA
\end{tabular} & $\begin{array}{l}\text { Análise dos resultados obtidos, } \\
\text { autorregistros no AVA. }\end{array}$ \\
\hline
\end{tabular}

Após a realização da intervenção pedagógica, foi preciso usar o método da avaliação para verificar se houve de fato avanços nas aprendizagens e se houve resultados em relação à forma como as estratégias foram trabalhadas e se essas promoveram aprendizagem (Damiani, Rochefort, Castro, Dariz, \& Pinheiro, 2014). Em outras palavras, foi preciso avaliar se a intervenção efetivamente foi planejada de modo a contribuir para avanços positivos na aprendizagem dos participantes da pesquisa. Para realizar a avaliação foram empregadas as técnicas de conversas diretas, perguntas detonadoras e entrevista semiestruturada. As conversas diretas e observações com os acadêmicos participantes foram registradas e gravadas, o que permitiu, a partir da observação e do registro, verificar como eles planejavam, o que priorizavam, como sistematizam as tarefas dentro de contexto da proposta sugerida pela intervenção da pesquisa. Nas conversas, muitas perguntas foram feitas: "como?", "o quê?", "onde?", "quando?", "por quê?". Todas as respostas decorrentes desses questionamentos são consideradas por 
Zimmerman (2009) graus de liberdade para tomada de decisão.

Foram duas as perguntas detonadoras apresentadas aos participantes: a) "Como você percebe o semestre em relação ao que foi realizado nesta intervenção, contendo três momentos pontuais: início - planejamento, desenvolvimento - execução e final - avaliação?"; b) "Como você atuou nesses três momentos? - destacando se houve alguma diferença em relação a momentos acadêmicos anteriores". Os relatos permitiram compreender como os estudantes percebiam o trabalho realizado e se redimensionavam suas ações e suas práticas. Todas essas informações contribuíram para que o pesquisador, na hora de fazer a análise dos dados, pudesse entender os pontos decisivos que emergiram dos relatos dos sujeitos da pesquisa (Creswell, 2010).

A entrevista semiestruturada mostrou ser uma possiblidade extremamente potente para verificar os processos de aprendizagens dos estudantes em relação à ARA e ao BIM. No campo da educação e da pesquisa qualitativa, a entrevista tem sido um meio poderoso com a qual o pesquisador consegue investigar como os estudantes compreendem o que fazem ou fizeram, isto é, como revelam o fenômeno no qual estão atuando ou estão envolvidos (Amado \& Ferreira, 2014). Para o estudo de caso, as entrevistas são de fundamental importância e podem ser conduzidas de forma espontânea, pois permitem que se dialogue com a pessoa, solicitando sua opinião sobre determinados fatos (Yin, 2014). O quadro 2 apresenta o guia da entrevista semiestruturada.

Quadro 2 - Guia da entrevista semiestruturada

\begin{tabular}{|l|l|}
\hline Blocos temáticos & Objetivos \\
\hline Legitimação & $\begin{array}{l}\text { Legitimar a entrevista (aspectos } \\
\text { éticos); Motivação }\end{array}$ \\
\hline $\begin{array}{l}\text { Sobre o ato de } \\
\text { projetar dos } \\
\text { estudantes }\end{array}$ & $\begin{array}{l}\text { Identificar o conhecimento que o } \\
\text { estudante tem sobre o ato de projetar e } \\
\text { emprego de estratégias }\end{array}$ \\
\hline $\begin{array}{l}\text { Sobre o ato de } \\
\text { modelar em BIM }\end{array}$ & $\begin{array}{l}\text { Identificar o conhecimento sobre } \\
\text { tecnologia BIM }\end{array}$ \\
\hline $\begin{array}{l}\text { BIM no Projeto } \\
\text { Arquitetônico }\end{array}$ & $\begin{array}{l}\text { Identificar se o uso de tecnologia BIM } \\
\text { contribui para uso de estratégias } \\
\text { autorregulatórias ao projetar }\end{array}$ \\
\hline $\begin{array}{l}\text { Finalização da } \\
\text { entrevista }\end{array}$ & $\begin{array}{l}\text { Promover a continuidade da utilização } \\
\text { de entrevistas }\end{array}$ \\
\hline
\end{tabular}

A terceira e última coleta de dados teve ênfase na aplicação do instrumento Escala de Autorregulação da Motivação para a Aprendizagem (EAMA) desenvolvida por Paulino, Lopes da Silva e Sá (2016), com o objetivo de identificar quais estratégias de autorregulação da motivação para a aprendizagem os estudantes do curso de Arquitetura e Urbanismo utilizam com mais frequência nas atividades de projeto e, se ao longo da pesquisa demonstraram envolvimento nas tarefas acadêmicas e na sua realização em relação a essas tarefas (Wolters, 2003; Wolters, Denton, York, \& Francis, 2014). O instrumento original recebeu adaptações de pesquisadores brasileiros e portugueses para ser aplicado em outros contextos educacionais da educação superior (Veiga Simão, Frison, \& Nonticuri, 2015).

Optamos por utilizar a adaptação feita por Veiga Simão, Frison e Nonticuri (2015) que reduziu a escala para 30 itens. Essa redução manteve o objetivo original da escala de Paulino, Lopes da Silva e Sá (2012), destinada a avaliar as crenças e estratégias utilizadas pelos estudantes para iniciar, manter e reforçar seu nível de motivação durante uma tarefa de aprendizagem. A EAMA de Veiga Simão, Frison e Nonticuri (2015) é composta por oito categorias as quais compreendem de três a quatro estratégias, cada uma conforme quadro 3 .

Quadro 3 - Escala de Autorregulação da Motivação para a Aprendizagem (EAMA)

\begin{tabular}{|l|c|}
\hline Estratégias de AMA & $\begin{array}{c}\mathbf{N}^{\circ} \text { de } \\
\text { questões }\end{array}$ \\
\hline Regulação do Interesse Situacional & 4 \\
\hline Aproximação a resultados positivos & 4 \\
\hline $\begin{array}{l}\text { Regulação pela aplicação de } \\
\text { Autorreforços }\end{array}$ & 3 \\
\hline $\begin{array}{l}\text { Regulação pela Estruturação do } \\
\text { Contexto }\end{array}$ & 3 \\
\hline Regulação pelo Valor da Tarefa & 4 \\
\hline $\begin{array}{l}\text { Regulação das Metas de Resultado - } \\
\text { Evitamento de resultados negativos }\end{array}$ & 4 \\
\hline $\begin{array}{l}\text { Regulação das Metas de Aprendizagem } \\
\text { Regulação pelas Crenças de } \\
\text { Autoeficácia }\end{array}$ & 4 \\
\hline Total de questões & 30 \\
\hline
\end{tabular}

Fonte: Adaptado de Veiga Simão; Frison; Nonticuri (2015)

\section{AUTORREGULAÇÃO DA MOTIVAÇÃO PARA A APRENDIZAGEM}

Autores como Wolters (2011), Paulino e Lopes da Silva (2012), Paulino, Sá e Lopes da Silva (2016) destacam a importância dos fatores relativos ao aspecto motivacional da autorregulação da aprendizagem, explicando que a capacidade dos aprendizes em regular sua motivação impacta no desempenho. Durante as intervenções ficou evidente o cuidado necessário com o planejamento das práticas que envolvam o uso de ferramentas BIM. Observou-se que dadas as posturas reveladas pelos estudantes e a valorização que dão à habilidade da modelagem em BIM, eles podem enfrentar insatisfação e ansiedades severas quando os resultados forem adversos. Foi possível também entender alguns processos da dimensão motivacional da aprendizagem autorregulada de BIM.

Ficam muito claras as metas de aprendizagem voltadas a aprendizagem de BIM e os desdobramentos positivos para outras áreas de conhecimento como, por exemplo, quando aplicado ao projeto arquitetônico. O contexto imediato de interesse apresenta para os estudantes a perspectiva de trabalhar com uma tecnologia de ponta. O uso por parte de estudantes mais experientes, juntamente com um ambiente dotado de um universo de páginas, blogs e canais de mídia divulgando e tirando dúvidas, mantém o interesse nas ferramentas e suas possibilidades. Em determinado ponto o interesse passa a ser individual, começa a ter características relativamente duradouras e o estudante se engaja em cursos extras ou on-line. Com interesse aumenta a autorregulação da aprendizagem e se revela potente quando os estudantes mostram que se engajam em tarefas de projeto ou atividades como projetos de ensino, pesquisas, oficinas, etc., mesmo que isso não Ihes seja exigido e, pelo contrário, encontre oposição no seu uso em diversos momentos. Nesse contexto entendemos que os estudantes estabelecem suas metas 
de aprendizagem, e a realização de um bom trabalho em BIM se apresenta como uma satisfação pessoal oriunda de um autojulgamento autoavaliativo positivo. Zimmerman (2002) destaca que a satisfação pessoal depende de os aprendizes alcançarem as metas estabelecidas, sendo isso fundamental para a perseverança e os esforços para aprender.

Foi visto que o papel da modelagem em BIM aumenta as crenças de autoeficácia sobre o domínio da habilidade acadêmica de projetar, mas apenas em determinados aspectos dessa prática, tal como o aspecto estrutural conforme apresentado pelos participantes da pesquisa. 0 aumento dessas crenças de autoeficácia auxilia na formação do senso de agência pessoal sobre a continuidade dos esforços de aprendizagem autorregulada na busca do atingimento de metas.

Os estudantes treinados exibem percepções mais positivas da eficácia específica da tarefa e são mais propensos a atribuir um desempenho bem-sucedido a fontes autorreguladas, como o uso de estratégias (Zimmerman B. J., 2000). Quando os estudantes precisam desenvolver suas habilidades de autorregulação, muitos se veem com dificuldades para realizar a transição entre o ensino médio, com as técnicas cognitivas desenvolvidas durante os anos escolares, e para atender às tarefas menos estruturadas, como o desenvolvimento do projeto arquitetônico, típicas do ambiente universitário (Pinho, Bastos, Dourado, \& Ribeiro, 2013). É de se considerar a possibilidade de desenvolver estudantes ingressantes em suas capacidades cognitivas e estratégias para que superem limitações de desenvolvimento em suas autoavaliações, autorregulação e transferência de estratégias de aprendizagem.

\section{CONCLUSÕES}

O software BIM, como regulador da aprendizagem, atua como uma orientação à meta de aprendizagem, nesse sentido, a autorregulação potencializa a capacidade de reforçar o feedback auto-orientado durante o processo. $\mathrm{O}$ investimento em uma metodologia de trabalho em BIM, envolve conhecimentos sobre como e por que escolher uma estratégia específica, o que foi relatado pelos participantes da pesquisa. Um fato positivo pois, só temos um contexto de autorregulação da aprendizagem quando um aprendiz se torna capaz de realizar avaliações sobre as direções que são tomadas para realizar os objetivos e, com isso, avaliar as eventuais mudanças na estratégia por ele selecionada (Bandura, 2008).

Ao longo dos depoimentos e da realidade contextual, percebemos que há um papel importante a ser preenchido, o do instrutor/professor, que serve de modelo para orientar os estudantes, de forma que possam estabelecer suas metas de aprendizagem. Assim, os estudantes podem concentrar esforços de aprendizagem focados no que pretenderam fazer com base na ferramenta. Esse processo atua como um regulador metodológico. Existe, porém, a necessidade de evitar que estudantes com baixas crenças de autoeficácia, recorram ao evitamento e estabeleçam metas de performance por atribuírem o sucesso ou insucesso a uma ideia subjacente fixa, ou seja, ter o "dom" de modelar, assim como existe a falsa crença no "dom" de projetar. É preciso estimular desde cedo, em disciplinas de projeto, que o uso gradual de ferramentas de tecnologia BIM contribuirá para que estudantes se tornem mais seguros e com a devida orientação utilizem mais estratégias de autorregulação.

Estudantes que foram estimulados a verificar suas modelagens e ignorar dificuldades, mantendo a perseverança, realizaram melhores modelos, pois pautamos nossa intervenção na crença em que ações sobre os processos de aprendizagem podem incidir sobre os pensamentos do estudante. $O$ investimento nesses pensamentos deve ser de tal forma que possibilitem aos aprendizes antecipar e avaliar os resultados das ações a realizar (componente metacognitiva), o que, assim, interage com a autoconsciência das suas expectativas de sucesso e das razões pessoais de valorização dos resultados a atingir (componente motivacional) (Paulino, Sá, \& Lopes da Silva, 2016).

\section{REFERÊNCIAS}

Zimmerman, B. J. (2002). Achieving Self-Regulation: The Trial and Triumph of Adolescence. En F. Pajares, F. Pajares, \& T. Urdan (Edits.), Academic Motivation of Adolescents (1 ed., Vol. 1, págs. 1-27).

Adamu, Z. A., \& Thorpe, T. (2016). How universities are teaching BIM: a review and case study from the UK. Journal of Information Technology in Construction (ITcon), v. 21, págs. p. 119-139.

Amado, J. (2014). Fundamentos da investigação qualitativa em educação. En J. Amado, Manual de investigação qualitativa em educação (2a ed., págs. 17-107). Coimbra, Portugal: Imprensa da Universidade de Coimbra. doi:http://dx.doi.org/10.14195/978-989-26-0879-2

Amado, J., \& Ferreira, S. (2014). A entrevista na investigação em educação. En J. Amado, Manual de investigação qualitativa em educação ( $2^{a}$ ed., págs. 207-290). Coimbra: Imprensa da Universidade de Coimbra. doi:http://dx.doi.org/10.14195/978989-26-0879-2

Ambrose, M. A. (2012). Agent Provocateur-BIM in the academic design studio. International Journal of Architectural Computing, v. 10, n. 1, págs. p. 53-66.

Arayici, Y., Coates, P., Koskela, L., \& Kagioglou, M. (2011). BIM adoption and implementation for architectural practices. Structural survey, 29(1), 7-25.

Avila, L. T. (2017). Autorregulação da aprendizagem no estágio de licenciatura em educação física: proposta formativa ancorada na pesquisa-ação. Pelotas: UFPel.

Bandura, A. (1999). Social cognitive theory: An agentic perspective. Asian Journal of Social Psychology, 2, 21-41.

Bandura, A. (2008). A evolução da teoria social cognitiva. En A. Bandura, R. Azzi, \& S. (. Polydoro, Teoria social cognitiva: conceitos básicos (págs. 15-41). Porto Alegre: Artned Editora.

Barison, M. B., \& Santos, E. T. (2013). Educational Activities for the Teaching-Learning of BIM. Proceedings of I BIM International Conference (BIC 2013).

Boruchovitch, E. (1999). Estratégias de aprendizagem e desempenho escolar: considerações para a prática educacional. Psicologia: Reflexão e Crítica, 12, págs. 361376. 79721999000200008

Boruchovitch, E. (set./dez. de 2014). Autorregulação da aprendizagem: contribuições da psicologia educacional para a formação de professores. Psicologia Escolar e Educacional, 18.

Brígitte, G. T., \& Ruschel, R. C. (out./dez. de 2016). Modelo de informação da construção para o projeto baseado em 
desempenho: caracterização e processo. Ambiente Construido, 16(4), 9-26. doi:http://dx.doi.org/10.1590/s167886212016000400102

Butler, D. L., Schnellert, L., \& Perry, N. E. (2017). Developing selfregulating learners. Toronto - Canadá: Pearson Canada Incorporated.

CBIC. (2016). Fundamentos BIM - Parte 1: Implantação. Brasília: Câmara Brasileira da Indústria da Construção.

Checcucci, É. d. (2014). Ensino-aprendizagem de BIM nos cursos de graduação em Arquitetura e Engenharia Civil. Encontro Da Associação Nacional De Pesquisa E Pós Graduação Em Arquitetura E Urbanismo, 3.

Checcucci, É. d., Pereira, A. P., \& Amorim, A. L. (Dezembro de 2013). Modelagem da Informação da Construção (BIM) no ensino de arquitetura. Proceedings of the 17th Conference of the Iberoamerican Society of Digital Graphics, 1(7), págs. $307-$ 311. doi:10.5151/despro-sigradi2013-0058

Chizzotti, A. (2003). A pesquisa qualitativa em ciências humanas e sociais: evolução e desafios. Revista portuguesa de educação, 16(2), págs. 221-236.

Creswell, J. W. (2010). Projeto de Pesquisa [recurso eletrônico] métodos qualitativo, quantitativo e misto (3 ed.). (D. d. Silva Ed., \& S. M. Rosa, Trad.) Porto Alegre: Penso. Recuperado e 2017 ,

https://integrada.minhabiblioteca.com.br/\#/books/978853632 $3589 /$

Damiani, M. F., Rochefort, R. S., Castro, R. F., Dariz, M. R., \& Pinheiro, S. S. (2014). Discutindo pesquisas do tipo intervenção pedagógica. Cadernos de Educação, 45, págs. 57-67.

Delatorre, V., Pereira, A. T., \& Miotto, J. (2015). BIM: Relatos de aplicação no ensino de arquitetura. Blucher Design Proceedings, 2(3), págs. 30-37.

Denzin, N., \& Lincon, G. (2003). Paradigmatic Controversies. Contradictions and.

Eastman, C., Teicholz, P., Sacks, R., \& Liston, K. (2014). MANUAL DE BIM: um guia de modelagem da informação da construção para arquitetos, engenheiros, gerentes construtores $e$ incorporadores ( $1^{a}$ edição ed.). (C. G. Ayres Filho, Trad.) Porto Alegre, RS, Brasil: Bookman.

Florio, W. (2007). Contribuições do Building Information Modeling no processo de projeto em arquitetura. Encontro de tecnologia da informaçao e comunicaçao na construçao civil, 3 .

Godoy, V. H., Cardoso, C. F., \& Borges, M. M. (2013). BIM: desafios para um conceito em construção no ensino de arquitetura e engenharia. En COBENGE, CONGRESSO BRASILEIRO DE EDUCAÇÃO EM ENGENHARIA (págs. 110). Gramado - RS. Recuperado el 16 de Fev de 2016, de https://www.fadep.br/engenhariaeletrica/congresso/pdf/118411_1.pdf

Jantzen, S. A. (2009). É possivel (aprender e ensinar a) projetar: projeto arquitetônico e urbanístico: orientações para o trabalho de curso (II ed.). (S. A. Jantzen, A. C. Silveira Jr., \& G. S. Fernandes, Edits.) Pelotas, RS, Brasil: Editora Universitária / UFPEL.

Kitchenham, B. A., \& Pfleeger, S. L. (2002). Principles of survey research: part 3: constructing a survey instrument. $A C M$ SIGSOFT Software Engineering Notes, 27(2), págs. 20-24.

Ledo, R. Z., \& Pereira, A. T. (2014). Objetos de Aprendizagem voltados ao ensino de modelagem digital 3D para arquitetura baseado no estilo cognitivo visual. Blucher Design Proceedings, 1(8), págs. 86-90

Machado, F. A., Ruschel, R. C., \& Scheer, S. (2016). Análise bibliométrica da produção brasileira de artigos científicos na área de BIM. Encontro Nacional De Tecnologia Do Ambiente Construido, 16.

Marques, S. (20-21 de June de 2013). Collaborative Intelligence in the Educational Context of BIM. BIC2013 - First BIM International Conference, 1, págs. 149-152.

Martínez, A. C. (2000). Ensaio sobre o projeto. (A. L. Spaltemberg, \& S. Fisher, Trads.) Brasília, DF, Brasil: Editora Universidade de Brasília.

Martinez-Pons, M., \& Zimmerman, B. (1990). Student differences in self-regulated learning: Relating grade, sex, and giftedness to self-efficacy and strategy use. Journal of educational Psychology, 82(1), pág. 51.

Moraes, R. (2004). Pensamento eco-sistêmico: educação, aprendizagem e cidadania no século XXI. Petrópolis, RJ: Vozes.

Moura, N. C., Giacaglia, M. E., \& Lara, A. H. (2013). BIM Implementation: An Experiment Integrating Practice, Theory and Didactic. En A. A. Costa, P. Couto, \& A. R. Meireles (Ed.), Building Information Modeling: A new construction paradigm (págs. 62-64). Porto: BIC 2013.

Nome, C. A., \& Queiroz, N. (2014). BIM: Processo e Integração no Ateliê de Projeto Arquitetônico. III ENAPARQ.

Palomera-Arias, R., \& Liu, R. (July de 2016). BIM laboratory exercises for a MEP systems course in a construction science and management program. (R. Amor, Ed.) Journal of Information Technology in Construction (ITcon), 21(13), págs. 188-203.

Paulino, P., \& Silva, A. L. (maio/junho/julho/agosto de 2012). Promover a regulação da motivação na aprendizagem. Cadernos de Educaçã, 42, 96-118. doi:HTTP://DX.DOI.ORG/10.15210/CADUC.V0I42.2150

Paulino, P., Sá, I., \& Lopes da Silva, A. (fev. de 2016). Crenças e estratégias da motivação na aprendizagem: Desenvolvimento de uma escala. Psychologic, 58(1), 65-87. Recuperado el 15 de mar. de 2018, de http://impactumjournals.uc.pt/psychologica/article/view/2755

Pedersen, S. A., \& Polydoro, S. A. (2017). Programas de Intervenção em Autorregulação da Escrita. En S. A. Polydoro, Promoção da autorregulação da aprendizagem: contribuições da Teoria Social Cognitiva (Vol. III, págs. 133-148). Porto Alegre: Letra1.

Pinho, A. P., Bastos, A. V., Dourado, L. C., \& Ribeiro, J. L. (2013). A transição do ensino médio para a universidade: um estudo qualitativo sobre os fatores que influenciam este processo $\mathrm{e}$ suas possiveis consequências comportamentais. XIII Coloquio de Gestión Universitaria. Obtenido de https://repositorio.ufsc.br/handle/123456789/25876

Pintrich, P. R. (2000). The role of goal orientation in self-regulated learning. En M. Boekaerts, M. Zeidner, \& P. R. Pintrich (Edits.), Handbook of self-regulation (págs. 451-502). Academic Press.

Polydoro, S. A., \& Azzi, R. G. (dez de 2009). Aprendizagem da autorregulação sob a perspectiva da teoria sócio-cognitiva: introduzindo modelos de investigação e intervenção. Psicol educ, 75-94. Recuperado el 2018 de julho de 22, de http://pepsic.bvsalud.org/scielo.php?script=sci_arttext\&pid=S 1414-69752009000200005\&lng=en\&nrm=iso

Rêgo, R. d., \& Carreiro, P. P. (2015). Fundamentos da gestão da informação projetual em curso de arquitetura e urbanismo. VII ENCONTRO BRASILEIRO DE TECNOLOGIA DE INFORMAÇÃO E COMUNICAÇÃO NA CONSTRUÇÃO (págs. 616-629). Porto Alegre: ANTAC; São Paulo: Blucher.

Romcy, N. M., Tinoco, M. B., \& Cardoso, D. R. (2015). A introdução do BIM em cursos de arquitetura e urbanismo: relato comparativo de duas experiências. En ENCONTRO BRASILEIRO DE TECNOLOGIA DE INFORMAÇÃO E 
COMUNICAÇÃO NA CONSTRUÇÃO (7. ed., págs. 513-525). 2015, Recife. Anais...Porto Alegre: ANTAC.

Rosário, P. S., Trigo, J., \& Guimarães, C. (2003). Estórias para estudar, histórias sobre o estudar: Narrativas Autoregulatórias na Sala de Aula. Revista Portuguesa de Educação, 16, 117133.

Rosário, P., Pereira, A., Högemann, J., Nunes, A. R., Figueiredo, M., Núñez, J. C., . . Gaeta, M. (2014). Autorregulación del aprendizaje: una revisión sistemática en revistas de la base SciELO. Universitas Psychologica, 2(13), 781-798.

Ruschel, R. C. (2014). TO BIM OR NOT TO BIM? III Encontro da Associação Nacional de Pesquisa e Pós-graduação em Arquitetura e Urbanismo , págs. 1-15.

Ruschel, R. C., Andrade, M. L., \& Morais, M. (abr./jun. de 2013). O ensino de BIM no Brasil: onde estamos? Ambiente Construido, 13(2), 151-165.

Santos, D. A., \& Alliprandini, P. M. (Dezembro de 2018). A promoção do uso de estratégias cognitivas em alunos do Ensino Médio. Psicologia Escolar e Educacional, 22(3), 535543.

Silveira, D. T., \& Córdova, F. P. (2009). A PESQUISA CIENTÍFICA. En D. T. [organizado por] Tatiana Engel Gerhardt, \& c. p.-U. de (Ed.), Métodos de Pesquisa (1 ${ }^{\text {a }}$ ed., págs. 31-42). Porto Alegre: Editora da UFRGS.

Souza, L. F. (2010). Estratégias de aprendizagem e fatores motivacionais relacionados. Educ. rev.(36), págs. p. 95-107. doi:10.1590/S0104-40602010000100008

Veiga Simão, A. M., \& Frison, L. M. (julho/agosto de 2013). Autorregulação da aprendizagem: abordagens teóricas e desafios para as práticas em contextos educativos. Cadern(45).

Veiga Simão, A. M., Frison, L. M., \& Nonticuri, A. R. (26 de 10 de 2015). Dos significados à autorregulação: perspectivas de estudantes com trajetórias acadêmicas de insucesso. Revista De Estudios E Investigación En Psicología Y Educación, Extr.(1). 048-053. https://doi.org/10.17979/reipe.2015.0.01.251

Vinšová, I., Achten, H., \& Matějovská, D. (2015). Integrating BIM in Education: Lessons Learned. Real Time-Proceedings of the 33rd eCAADe Conference, 127-131.

Williams, J. H. (2008). Employee engagement: Improving articipation in safety. . Professional Safety, , 12(53), 40-45.

Wolters, C. A. (2003). Regulation of Motivation: Evaluating an Underemphasized Aspect of Self-Regulated Learning. Educational Psychologist, 38:4, 189-205. doi:10.1207/S15326985EP3804_1
Wolters, C. A. (2011). Regulation of Motivation: Contextual and Social Aspects. Teachers College Record, 113(2), 265-283.

Wolters, C. A., Denton, C. A., York, M. J., \& Francis, D. J. (March de 2014). Adolescents' motivation for reading: group differences and relation to standardized achievement. Reading and Writing, 27, págs. 503-533. Recuperado el Dez de 2018, de https://link-springercom.ez66.periodicos.capes.gov.br/journal/11145

Wu, W., \& Issa, R. R. (2013). BIM Education and Recruiting: Survey-Based Comparative Analysis of Issues, Perceptions, and Collaboration Opportunities. Journal of professional issues in engineering education and practice, 140(2), pp. 04013014-1 / 04013014-5

Ximenes dos Santos, O. J., \& Boruchovitch, E. (2011). Estratégias de Aprendizagem e Aprender a Aprender: Concepções e Conhecimento de Professores. Psicologia Ciência e Profissão, 31(2), 284-295. Recuperado el 15 de 05 de 2018, de http://www.redalyc.org/articulo.oa?id=282021811007

Yin, R. K. (2014). Estudo de Caso Planejamento e Métodos (5 ed.). Bookman.

Zimmerman, B. J. (September de 1989). A social cognitive view of self-regulated academic learning. Journal of Educational Psychology, 81(3),, 329-339.

Zimmerman, B. J. (2000). Attaining Self-Regulation: A social cognitive perspective. En M. Boekaerts, M. Zeidner, \& P. R. Pintrich (Edits.), Handbook of Self-Regulation (Vol. 1, págs. 13-39). New York: Academic Press.

Zimmerman, B. J. (2002). Achieving Self-Regulation: The Trial and Triumph of Adolescence. En F. Pajares, F. Pajares, \& T. Urdan (Edits.), Academic Motivation of Adolescents (1 ed., Vol. 1, págs. 1-27).

Zimmerman, B. J. (Spring de 2002). Becoming a Self-Regulated Learner: An Overview. Theory Into Pratice, 41(College of Education, The Ohio State University), 65-70.

Zimmerman, B. J. (2011). Motivational Sources and Outcomes of Self-Regulated Learning and Performance. En Handbook of Self-Regulation of Learning and Performance Routledge (págs. 49-64). doi:10.4324/9780203839010.ch4

Zimmerman, B. J. (2013). From Cognitive Modeling to SelfRegulation: A Social Cognitive Career Path. Educational Psychologist, 48:3, 135-147. 